\title{
Competitividad de la empresa industrial: una reflexión metodológica
}

\author{
Alvarez Medina, Lourdes*
}

\section{Resumen}

En los últimos años han aparecido una infinidad de enfoques para estudiar la competitividad, sin embargo no existe consenso en la definición, dimensiones o categorías en que debe estudiarse. Este trabajo reflexiona sobre las limitaciones de los indicadores de competitividad más difundidos utilizand o para esto fuentes secundarias. Se revisan enfoques para evaluar la competitividad y se analizan estudios previos que evalúan la competitividad de la industria siderúrgica en México mediante indicadores de comportamiento exportador y de innovación tecnológica. Estos indicadores demostraron debilidades ya que una empresa que incrementa sus ventas en el mercado mundial puede no dejar beneficios al país o a las personas que en ella laboran, además las empresas más innovadoras no siempre son las más competitivas, lo que se relaciona con la estructura y características de la industria. Se concluye que la metodología para el análisis de la competitividad no puede estandarizarse, sino que es más bien un mosaico de posibilidades que debe analizarse para establecer los métodos que mejor ayuden a explicar la actuación de una empresa o industria.

Palabras clave: Enfoque competitivo, Industria siderúrgica, México.

\section{Competitiveness in the Industrial Enterprise: a Reflection on Methodology}

\begin{abstract}
In recent years, an immense number of approaches to studying competitiveness have appeared, nevertheless, no consensus exists regarding the definition, dimensions or categories that should be studied. This work takes a look at the weaknesses of most well-known competitiveness indicators using secondary sources. Approaches are reviewed to evaluate competitiveness, and previous studies evaluating the competitiveness of the steel industry in Mexico are analyzed using indicators of exporter behavior and technological innovation. These indicators demonstrate weaknesses because a company that increases sales in the world market may not bring benefits to the country or its employees. Also, the most innovating companies are not always the most competitive, a situation

Recibido: 08-10-07. Aceptado: 26-06-08

* Profesora titular de la División de Investigación, Facultad de Contaduría y Administración, Universidad Nacional Autónoma de México. E-mail: malvarez @correo.fca.unam.mx
\end{abstract}


Competitividad de la empresa industrial: una reflexión metodológica

Alvarez Medina, Lourdes

related to industry structure and characteristics. Conclusions are that the methodology for analyzing competitiveness cannot be standardized, but is rather a mosaic of possibilities that must be analyzed in order to establish methods that best help to explain the performance of a company or industry.

Key words: Competitive approach, steel industry, Mexico.

\section{Introducción}

A partir de los años ochenta se impulsó el modelo económico de libre mercado con economías abiertas y progresivamente desreguladas. Se privatizaron las empresas en manos del Estado, se promovió la inversión extranjera directa, se consolidaron las industrias a nivel global y regional, y se incrementó la competencia obligando a las empresas a buscar formas de mejorar su competitividad para poder conservar sus mercados (Rueda, 2000).

En las últimas décadas han aparecido una infinidad de métodos y enfoques para estudiar la competitividad, sin embargo no existe consenso en su definición, las dimensiones ni los niveles en que debe estudiarse, medirse y promoverse.

Como antecedentes de los estudios de competitividad, se menciona la teoría clásica de las ventajas comparativas de David Ricardo del siglo XVIII (citado en Instituto mexicano de ejecutivos de finanzas (IMEF), 1995; Krugman, 2000) que enfatizó que las naciones deben especializarse en aquellos sectores productivos en que tienen abundancia de recursos naturales y de factores de producción porque les permite tener costos de producción más bajos. Sin embargo, la teoría nunca explicó por qué los países tendrían niveles de productividad diferentes.

Posteriormente Heckscher y Ohlin argumentaron que las ventajas compara- tivas surgen de las diferencias en la dotación de factores de producción. Los productos difieren en su composición de capital y mano de obra mientras que los países difieren en su oferta de capital y mano de obra, por lo tanto hay que exportar bienes en los que estos factores permiten menores costos. Esta teoría fue criticada por autores que argumentaron que la innovación jugaba un papel importante e incluyeron en el análisis la demanda local y las economías de escala (IMEF, 1995). Sin embargo, cuando Leontinef (1985) realizó un estudio empírico previendo que Estados Unidos de América (EUA) debía exportar bienes intensivos en capital e importar bienes intensivos en trabajo descubrió que los bienes importados requerían $30 \%$ más de capital por trabajador que los bienes exportados lo que resultó ser opuesto al modelo de Heckscher y Ohlin.

En la década de los ochenta el trabajo de Porter (1991, 2000, 2003) analiza por qué algunas industrias son más atractivas que otras para el inversionista, las estrategias de una empresa con relación a sus rivales, las acciones estratégicas competitivas que pueden afectar el atractivo general de la industria, y la competitividad de la empresa utilizando la cadena de valor. Cuando presenta su modelo, a principio de los años noventa, para explicar la competitividad de las naciones enfatizando el papel de la estructura de la industria y la importancia de la acción del 
gobierno, se retoma el debate de las ventajas comparativas y da un nuevo impulso al estudio de la competitividad.

Diferentes autores han argumentado que no es posible estudiar la competitividad considerando únicamente medidas de desempeño como son: las exportaciones, la balanza de pagos o la productividad sino que es necesario medir factores que generan un potencial para competir como la tecnología, el acceso a materias primas, capital, mano de obra calificada, etc. y las habilidades administrativas que permiten cambiar el potencial competitivo en desempeño como son las aptitudes en mercadotecnia y en relaciones con la comunidad (Buckley et al.,1988).

El objetivo de este trabajo es reflexionar sobre las limitaciones de los indicadores de competitividad más difundidos. Se ejecuta una revisión de los diferentes enfoques para estudiar la competitividad y posteriormente se analiza un estudio que evalúa la competitividad de la industria siderúrgica mexicana después de su privatización (1992-2002). Esto permite argumentar porqué los enfoques más difundidos para medir la competitividad son débiles y la creciente necesidad de utilizar metodologías mixtas que incluyan métodos cualitativos y cuantitativos.

Este trabajo es de tipo descriptivo, documental, basado en fuentes de información secundarias; se consultaron estadísticas de la Cámara nacional del hierro y el acero (Canacero), tesis, artículos de revistas especializadas y libros. El estudio de competitividad de la industria siderúrgica que se analiza utiliza la metodología desarrollada por la Comisión Económica para América Latina (CEPAL, 1999); que se centra en el análisis de la dinámica de las exportaciones de un país en el mercado mundial y mide la innovación mediante el índice Índico (Corona, 1997) y estudios de caso.

\section{Importancia del estudio de la competitividad y sus diferentes enfoques}

La competitividad se ha convertido en una de las preocupaciones centrales de los gobiernos y los sectores productivos porque se ha relacionado con el crecimiento del ingreso, el empleo, la inversión y el comercio. También ha sido considerada como una estrategia para hacer frente a los cambios ocasionados por la apertura comercial, los ajustes estructurales y la reconversión productiva (Metcalf et al., 2002).

La importancia que la competitividad tiene para las empresas, los gobiernos y la comunidad académica es indiscutible; sin embargo, no existe consenso en su definición, las dimensiones ni los niveles en que debe estudiarse, medirse y promoverse (Buckley et al., 1988; Krugman, 1997; Cellini y Soci, 2002; Ganzaroli et al., 2008). A continuación se presentan las propuestas para el estudio de competitividad por niveles y en cada uno se incluyen diferentes definiciones de competitividad.

\subsection{El estudio de la competitividad a nivel de países}

Tradicionalmente la competitividad de un país se había explicado a través de la teoría clásica de ventajas comparativas, la cual pone énfasis exclusivamente en la abundancia de los recursos naturales y de 
Competitividad de la empresa industrial: una reflexión metodológica

Alvarez Medina, Lourdes

los factores de producción. Esta teoría resultó ser contradictoria, ya que supone competencia perfecta cuando en realidad la competencia es fundamentalmente imperfecta; tanto empresas como gobiernos pueden actuar sobre los flujos comerciales y, por lo tanto, modificar la competencia de una nación. Debido a la naturaleza imperfecta de la competencia en los mercados -predominio de oligopolios, monopolios, oligopsonios, monopsoniossurgieron nuevas explicaciones acerca de la competitividad. Algunos la entienden como un fenómeno macroeconómico, otros argumentan que depende de la disposición de la fuerza de trabajo barata y abundante, otras teorías aducen la presencia de un intercambio desigual entre el "centro" y la "periferia" (IMEF, 1995).

Porter (1991) propuso estudiar la competitividad de una nación analizando patrones de éxito competitivo en las industrias y usando las exportaciones sostenidas a un amplio conjunto de otros países y/o salidas significativas de inversión extranjera, basada en habilidades y activos creados en el país de origen como indicador. Su teoría sugiere que los atributos de una nación moldean el entorno económico, de tal forma que estos promueven o impiden la creación de ventajas competitivas sostenibles en el largo plazo. El marco en el que se gestan las ventajas competitivas consta de cuatro atributos: a) La condición de los factores (mano de obra y capital) se concibe de un modo dinámico y la escasez de factores se concibe como fuente generadora de ventajas competitivas que propicia la innovación. b) La demanda local sofisticada es importante ya que representa un incentivo para desarrollar nuevos produc- tos con mayor rapidez, c) Proveedores competitivos internacionalmente benefician a las empresas locales. Si logran la formación de clusters, estos tienen efectos multiplicadores, ya que las industrias complementarias como servicios, comparten actividades dentro de la cadena de valor, d) La estructura de la industria y la intensidad de la rivalidad que influyen en las utilidades promedio de la industria.

Porter rechaza una definición puramente macroeconómica de competitividad y señala que el déficit presupuestario, las tasas de interés o la abundancia de la mano de obra no son conceptos significativos de competitividad. Por el contrario, la tasa de crecimiento de la productividad es determinante del nivel de vida a largo plazo de un país (Abdel y Romo, 2004).

De acuerdo con Krugman (1997) la definición más popular en los últimos años es la proporcionada por el Competitiveness Policy Council de los Estados Unidos que señala "la competitividad se refiere a la habilidad de una economía nacional para producir bienes y servicios que superan las pruebas de los mercados internacionales, al mismo tiempo que los ciudadanos pueden tener un estándar de vida creciente y sustentable en el largo plazo".

La Organización para el Crecimiento Económico y el Desarrollo (OCED) señala que "Competitividad es el grado en que un país puede, en condiciones de libre mercado y justa competencia, producir bienes para pasar las pruebas de los mercados internacionales, y al mismo tiempo mantiene o expande los ingresos reales de la población en el largo plazo" (Villareal, 2003: 187).

La Comisión Económica para América Latina y el Caribe, señala que "Com- 
petitividad es el proceso de expansión de la oferta exportable y penetración de mercados externos con el consecuente mejoramiento en el nivel de vida de la población" (Villareal, 2003:187).

Estas definiciones tienen en común las exportaciones y el nivel de vida de la población lo que parece razonable. Sin embargo (Krugman, 1997:18) discute la relación entre el estándar de vida de la población y la capacidad de producir bienes que compiten en el mercado internacional y señala que "en una economía con poco comercio internacional, el crecimiento en estándares de vida está determinado por factores domésticos, principalmente la tasa de crecimiento de la productividad". En consecuencia, el estándar de vida de la población dependería de la productividad y no tendría nada que ver con la competencia internacional. En este sentido, Krugman menciona que "Aún cuando el comercio mundial es más intenso que antes, los estándares de vida están determinados por factores domésticos más que por la competencia por los mercados mundiales. Parte de la respuesta a esta polémica es que el mundo no es tan interdependiente como uno podría pensar. Aún ahora, las exportaciones de Estados Unidos son sólo el 10\% de su Producto Nacional Bruto (PNB); por lo tanto es una economía que produce bienes y servicios para su propio uso" (Ramírez y Wallace, 1998:17).

Krugman (1997:18) consideró que es más problemático definir la competitividad de una nación que la de una empresa, "cuando decimos que una empresa no es competitiva, queremos decir que su posición es insostenible, al menos que mejore su desempeño dejara de existir.
Los países por otro lado no cierran, pueden ser felices o infelices con su situación económica pero no tienen una línea de flotación definida". Los países no compiten entre sí más que por la inversión extranjera directa, por lo que evaluar la salud económica de un país y calificarla como competitividad es inadecuado.

Diversas instituciones como el World Economic Forum (WEF) y el Banco Mundial (BM), entre otros, han formulado índices de competitividad que buscan clasificar a los países utilizando varias dimensiones y asignándoles valores que al ser sumados integran un indicador que permite comparar el desempeño de los países.

EI WEF (2006) evalúa 125 países, formando el índice con tres dimensiones: factores básicos, factores de eficiencia y factores de innovación; estos factores se evalúan con base en información estadística y usando encuestas de opinión. a) Los factores básicos incluyen: instituciones, marco económico, salud y educación b) Los factores de eficiencia incluyen: educación superior y capacitación, eficiencia de mercado y esfuerzo tecnológico c) Los factores de innovación incluyen: sofisticación de las empresas e innovación (http://www3.weforum.org/ consultado en octubre de 2007).

El Reporte sobre el Progreso Económico y Social en América Latina (BID, 2001, citado en Abdel y Romo, 2004:59) define la competitividad como "la calidad del entorno para realizar inversiones y para incrementar la productividad en un clima de estabilidad macroeconómica e integración a la economía nacional". Este reporte pone énfasis en identificar las deficiencias en los mercados de los factores de producción 
Competitividad de la empresa industrial: una reflexión metodológica

Alvarez Medina, Lourdes

que limitan el desarrollo del sector privado y que pueden ser corregidos mediante la implementación de políticas públicas. Entre los factores incluidos están: el crédito, los recursos humanos, la infraestructura portuaria, el suministro de electricidad y las telecomunicaciones y las tecnologías de la información.

Sobre estos indicadores, se ha dicho que los fundamentos teóricos resultan problemáticos, las definiciones utilizadas son muy amplias, el enfoque es sesgado, la metodología es errónea y las mediciones cualitativas son vagas, repetitivas y mal formuladas (Lall, 2000, 2003). Aún así, su uso se ha generalizado y los indicadores han sido importantes en la toma de decisiones de los inversionistas.

\subsection{El estudio de la competitividad a nivel de la industria}

Porter (2000) propone estudiar la competencia en una industria analizando cinco factores: rivalidad de los competidores, poder de negociación de los proveedores, poder de negociación de los clientes, amenaza de nuevas empresas entrantes y amenaza de productos sustitutos. Además, describe cuatro tipos de industrias -maduras, emergentes, fragmentadas y en declinación- y tres estrategias genéricas -bajo costo, diferenciación y enfoque- a las que se apegan la mayor parte de las empresas en una industria. Su metodología se critica porque no hay una lógica que sustente la selección de los cinco factores; a pesar de que Porter señala que llegó a esta conclusión después de estudiar cientos de empresas y no hay argumentos que apoyen está generalización (Aktouf, 2004).
La Comisión Económica para América Latina y el Caribe (CEPAL), (1999) propone analizar la competitividad de la industria de cada país con base en su comportamiento exportador. Esta metodología permite conocer el desempeño de una industria o producto que está siendo exportado al mercado internacional. También, ayuda a detectar a los países que compiten en una industria determinada y a conocer la dinámica o el comportamiento de las exportaciones. Esta es una excelente herramienta para comprender la forma de competencia en mercados internacionales a través del comportamiento exportador de los países y de las industrias. Cuando se realiza este análisis es conveniente recordar que La Conferencia de las Naciones Unidas sobre Comercio y Desarrollo enfatiza que la competitividad en las exportaciones comienza con un incremento en la participación de mercado, también implica diversificar la canasta de exportación, sostener tasas más elevadas de crecimiento en la exportaciones, aumentar el contenido tecnológico y de habilidades en las actividades de exportación y ampliar la base de empresas locales capaces de competir internacionalmente, de tal manera que la competitividad se vuelva sustentable y venga acompañada de ingresos crecientes (UNCTAD, 2002, citada en Abel y Romo, 2004).

\subsection{El estudio de la competitividad a nivel de la empresa}

Una empresa es competitiva si puede producir productos y servicios de calidad superior y costo más bajo que sus competidores domésticos e internacionales. La competitividad es sinónimo de un desempeño redituable a largo plazo, la 
habilidad para compensar a sus empleados y proveer retornos sobre la inversión superiores al promedio a sus accionistas (The Aldington report, 1985, citado en Buckley et al., 1988).

En el nivel microeconómico la competitividad se refiere a la habilidad de una empresa de crecer en tamaño, participación de mercado y rentabilidad. Tradicionalmente los costos de producción determinaban una competitividad relativa pero otros factores se han ido mostrando importantes como son: habilidades y motivación de la fuerza de trabajo, capacidades de investigación y desarrollo, habilidades para adoptar nuevas tecnologías, factores organizacionales y administrativos, relaciones con otros actores como clientes, proveedores, otras empresas, etc. (Clark y Guy, 1998).

Polevnsky (2003:177) señala que "la competitividad a nivel empresa es la capacidad para vender más productos o servicios y mantener o aumentar su participación en el mercado sin necesidad de sacrificar utilidades o salarios, o dañando el ambiente social o natural".

La participación de mercado es el elemento común en las definiciones y la rentabilidad o participación lucrativa, los salarios de los trabajadores y el cuidado al ambiente son elementos de competitividad.

Coriat (1998), citado en Simón 2002), presenta una noción compleja de competitividad que incluye múltiples factores de "costo" y de "no costo". Los primeros incluyen el costo del trabajo que comprende los salarios directos e indirectos; el costo de capital, formado por las tasas de interés y por las tasas de ocupación de los equipos y calidad del trabajo; los precios de los insumos; y las econo- mías de escala para reducir costos. Entre las dimensiones de "no costo" propone el margen de ganancia de las empresas; la calidad de los productos y de los servicios; la capacidad instalada; la capacidad de adaptarse a normas técnicas y de diferenciación de productos; tiempos de entrega; especialización hacia los mercados de demanda creciente; y eficacia de las redes. Coriat marca una relación de la empresa con la industria al seleccionar factores como redes interempresariales y tiempo de entrega "justo a tiempo".

Porter (2003) propone analizar la competitividad de la empresa a través del análisis de la cadena del valor. Este modelo intenta detectar las fuentes que pueden generar una ventaja competitiva, al examinar las actividades que una empresa desarrolla. El análisis incluye dos dimensiones: actividades primarias y actividades de apoyo. Las actividades primarias a su vez se dividen en cinco áreas de análisis: logística de entrada, operaciones, logística de salida, mercadotecnia y ventas y servicio. Las actividades de apoyo se dividen en cuatro áreas de análisis: adquisiciones, desarrollo tecnológico, administración de recursos humanos e infraestructura de la empresa. Porter no indica porqué seleccionó estas actividades como críticas en la cadena de valor, dejando fuera el área de finanzas que es esencial en el funcionamiento de la empresa. Aktouf (2004) le critica porque no incluye factores como cultura corporativa, moral del trabajador, nivel de comunicación, trabajo en equipo, liderazgo y habilidades interpersonales para traer al máximo el potencial de los trabajadores. Estos factores son más difíciles de analizar y modificar que los señalados en la 
Competitividad de la empresa industrial: una reflexión metodológica

Alvarez Medina, Lourdes

cadena de valor, aunque también son fuentes de ventaja competitiva.

Prahalad y Hamel (1990) señalan que existen competencias centrales que dan a la empresa ventajas competitivas, pero indican que éstas son diferentes para cada empresa. Por su parte, la teoría basada en recursos incluye entre las variables que se identifican como una ventaja competitiva: la calidad total, la tecnología, la administración del conocimiento, la innovación y principalmente, el elemento humano (Aktouf, 2004).

Castañon (2003) propone que la competitividad es una función que resulta de seis dimensiones dentro de la empresa: a) Empresarial; identifica la forma en que se lleva a cabo la planeación estratégica, b) Estrategia y gestión financiera; analiza la planeación respecto a los recursos financieros, c) Mercado; analiza las decisiones de la empresa respecto al mantenimiento y expansión de los mercados actuales, así como el acceso a nuevos mercados, d) Tecnología; identifica las relaciones entre la estrategia tecnológica y la estrategia competitiva, e) Administración de operaciones; la forma en que la empresa coordina los insumos con el equipo y los recursos humanos para la manufactura eficiente de los productos, f) Cómputo y tecnología de la información: actividades asociadas con el uso y actualización de equipo de cómputo y programas para llevar a cabo diversas tareas en distintas áreas de la organización. Estas dimensiones se miden en varias empresas; después los resultados de una empresa se comparan con el promedio de empresas analizadas. Es una propuesta útil que justifica haber seleccionado estas dimensiones después de aplicar el instrumento en dos investigaciones anteriores y coincide con la propuesta de Porter en tres de las dimensiones: mercadotecnia, tecnología y operaciones.

\subsection{Estudio de la competitividad en varios niveles}

Esser et al. (1996) proponen un análisis sistémico de la competitividad que incluye cuatro niveles: meta, macro, meso y micro. a) en el nivel meta se habla de la capacidad del Estado para conducir la economía y de patrones de organización social, política y económica que permiten incentivar la creatividad. Se debe tener una administración pública con visión a largo plazo y una iniciativa privada que incluya una visión de desarrollo sustentable y no únicamente enfocada a la ganancia b) en el nivel macro se evalúa la capacidad para asegurar la eficiencia de mercados y para asignar eficazmente recursos, por lo que se necesita un contexto económico, político y jurídico estable, como son políticas monetaria, fiscal, comercial, cambiaria y de competencia c) el nivel meso estudia las políticas sectoriales y las instituciones de fomento específicas, como son la política tecnológica, la educación, la formación profesional, la regulación ambiental y la infraestructura, que son elementos de competitividad d) el nivel micro considera la innovación, el capital humano calificado y las redes de colaboración eficientes. La cadena de valor, la subcontratación y los clusters son la interfase entre el nivel de la empresa y el de la industria.

De acuerdo con Villareal (2003), existe una interacción dinámica entre varios sistemas que afecta el desempeño competitivo de las empresas: a) el sistema, definido por el grado de apertura 
económica y exposición a la globalización; b) el sistema macroeconómico, que define el ritmo de crecimiento y las condiciones de financiamiento e inversión, c) el sistema sectorial industrial, que define la densidad y solidez del tejido productivo de un país, estado o región, d) el sistema institucional, que define las reglas bajo las cuales funcionan los mercados y opera la economía en su conjunto, lo mismo que el Estado y e) el sistema microempresarial, que es el núcleo base de la competitividad. El funcionamiento de cualquiera de estos sistemas afecta a los demás de manera directa. El discernimiento correcto de la competitividad o del crecimiento competitivo requiere del análisis de la interacción dinámica entre estos sistemas.

Dussel (2003) señala que desde la década de los noventa, diversos autores (Esser et al., 1996; Messner y Meyer-Stamer, 1999; Meyer-Stamer, 2000) desarrollaron el concepto de competitividad sistémica en contraposición al presentado por Porter y al de competitividad estructural de la OCDE, pero asevera que es en el nivel meso económico en donde se generan las ventajas competitivas.

Finalmente se concluye que la competitividad es un concepto dinámico, evolutivo y multidimensional, que puede evaluarse en diferentes niveles y dimensionese incluye sistemáticamente la comparación con los rivales. Los niveles pueden incluir un país, la industria, una región o una empresa. Las dimensiones pueden cambiar dependiendo del enfoque, pero existen dos variables que son mencionadas constantemente en la literatura: la habilidad para exportar y el uso eficiente de los factores de producción, que repercute directamente sobre la productividad.

\section{Competitividad de la industria siderúrgica en México}

En este apartado se presenta una investigación sobre competitividad en la industria siderúrgica mexicana y a partir de ésta se forma una discusión sobre la estructuración de metodologías para el estudio de la competitividad.

La investigación sobre la industria siderúrgica buscó determinar si a partir de su privatización, a principio de la década de los noventa, la industria siderúrgica había incrementado su competitividad. Se exploró como hipótesis central que "La industria siderúrgica integrada en México había incrementado su competitividad, entre 1992 y 2002, ganando participación en el mercado internacional mediante nuevas estrategias tecnológicas que le permitieron fabricar productos con mayor valor agregado" (Alvarez, 2000: 155; 2002:207).

Las siderúrgicas estudiadas fueron cuatro y eran responsables del $87 \%$ de la producción de acero en México: a) Altos Hornos de México (AHMSA), b) Siderúrgica Lázaro Cárdenas las Truchas (SICARTSA) que se dividió al privatizarse quedando dos empresas: Sicartsa e ISPAT Mexicana (IMEXA) c) Hojalata y lámina de México (HYLSAMEX) era privada.

Para alcanzar el objetivo se desarrolló la siguiente metodología: 1) se analizó la competitividad de la industria siderúrgica con base en su comportamiento exportador con la metodología desarrollado por la Comisión Económica para América Latina y el Caribe 2) se evaluó la posición competitiva de cada empresa calculándose el índice Indico 
Competitividad de la empresa industrial: una reflexión metodológica

Alvarez Medina, Lourdes

de innovación 3) se analizó hacia dónde se dirigió la inversión en tecnología en cada una de las cuatro siderúrgicas integradas en México y 4) se analizó el cambio en la mezcla de productos a nivel nacional y por empresa.

En la revisión general de la literatura se encontró que la Siderúrgica era una industria madura a nivel mundial con las siguientes características: $30 \%$ de exceso de capacidad instalada e inversiones en nuevas plantas anunciadas, altas barreras de salida, precios a la baja, la lámina rolada en frío había bajado $2.2 \%$ entre 1984 y 2000 , productos con baja diferenciación, considerados una mercancía, crecimiento mínimo de la demanda, 0.8\% entre 1990 2000 y alta rivalidad (Sanahan, 2002).

\subsection{Metodología para evaluar la competitividad de la industria siderúrgica basada en el análisis de las exportaciones}

Para evaluar la competitividad de la industria siderúrgica se analizó la dinámica de la participación de las exportaciones de un país en el mercado mundial. Más del $80 \%$ de las exportaciones de acero se enviaban a Estados Unidos por lo que se considera como el socio comercial. Los indicadores de desempeño que se utilizan son los siguientes:

$\mathrm{Xi}=$ valor de las exportaciones siderúrgicas de México al socio comercial.

$\mathrm{Yi}=$ valor de las exportaciones totales de México al socio comercial.
M1 =valor total de importaciones siderúrgicas del socio comercial.

M2 =valor total de las importaciones del socio comercial.

Una vez calculados los indicadores, el desempeño exportador se puede calificar de acuerdo con la siguiente tipología:

- Estrella creciente son rubros en los que la tasa de crecimiento de las importaciones siderúrgicas crece más que la tasa de las importaciones totales. Es decir, el producto crece en un mercado dinámico.

- Estrella menguante: las importaciones siderúrgicas crecen menos que el total de las importaciones pero el producto mexicano aumenta su participación. Es decir el producto crece en un mercado que pierde dinamismo.

- Oportunidades perdidas: las importaciones siderúrgicas crecen más que el promedio, pero el producto mexicano es desplazado por producto de otros países competidores o sea que se pierde participación.

- En retirada: Son rubros estancados en las que no crecen las importaciones siderúrgicas ni el producto mexicano.

En el análisis efectuado con el MA$\mathrm{GIC}^{1}$ se encontró que la industria siderúrgica incrementó sus exportaciones a Estados Unidos de América (EUA) entre 1992 y 2002 , pasando de 2.91 a $9.58 \%$ en el capítulo (72) hierro y acero (Ver Tabla 1).

1 Para llevar a cabo el análisis se utilizó el banco de datos MAGIC (Modulo para Analizar el Crecimiento del Comercio Internacional), desarrollados por CEPAL. 
Tabla 1

Exportaciones de la industria siderúrgica mexicana hacia Estados Unidos

Grupo 72 del sistema armonizado hierro y acero

\begin{tabular}{cccc}
\hline Año & $\begin{array}{c}\text { Participación en } \\
\text { importaciones } \\
\text { siderúrgicas de } \\
\text { EUA = X1/M1 }\end{array}$ & $\begin{array}{c}\text { Estructura de } \\
\text { Exportación de México } \\
=\text { X1/Y1 }\end{array}$ & $\begin{array}{c}\text { Estructura de } \\
\text { importaciones } \\
\text { siderúrgicas de EUA } \\
=\text { M1/M2 }\end{array}$ \\
\hline 1992 & 2.91 & .66 & 1.50 \\
1993 & 3.92 & .84 & 1.47 \\
1994 & 4.69 & 1.17 & 1.86 \\
1995 & 6.91 & 1.8 & 1.65 \\
1996 & 7.55 & 1.36 & 1.66 \\
1997 & 8.3 & 1.32 & 1.57 \\
1998 & 6.49 & 1.11 & 1.78 \\
1999 & 8.02 & .94 & 1.26 \\
2000 & 7.28 & .79 & 1.21 \\
2001 & 7.64 & .61 & .92 \\
2002 & 9.58 & .82 & .99 \\
\hline
\end{tabular}

Elaborado con el banco de datos MAGIC "Sistema par analizar el crecimiento del comercio internacional" CEPAL, México, agosto de 2002.

La siderúrgica mexicana estaba incrementando su participación en un mercado que perdía dinamismo. Esto se puede ver en la tercera columna, que indica que la estructura de importaciones siderúrgicas de EUA perdió dinamismo pasando de 1.50 a $.99 \%$-entre 1992 y 2002. Es por esto que el grupo 72 es evaluado como estrella menguante: en el periodo bajo estudio las exportaciones siderúrgicas de México aumentan en un mercado que pierde dinamismo).

Por otro lado, el valor de las exportaciones siderúrgicas de México a EUA sobre el total de las exportaciones de México a EUA se conoce como la estructura de exportación de México esto se puede ver en la segunda columna. El peso de los productos siderúrgicos en el total de las exportaciones ha variado incrementado su participación al pasar de .66 a 1.36\% en 1996 y disminuyendo posteriormente a $82 \%$ en 2002 . La disminución entre 1996 y 2002 se debe a que a partir de la crisis de 1995 las exportaciones totales de México se diversifican y otras industrias como la automotriz adquieren mayor relevancia en la mezcla de productos exportados. Sin embargo, se puede decir que cada vez son más importantes las exportaciones siderúrgicas para México.

\subsection{Productos siderúrgicos y exportaciones}

Después del análisis general fue necesario saber cuáles eran los principa- 
Competitividad de la empresa industrial: una reflexión metodológica

Alvarez Medina, Lourdes

les productos que se exportaban a Estados Unidos y si estos eran productos con alto valor agregado México fabrica cinco grupos básicos de productos los cuales se describen de menor a mayor valor agregado: a) los semiterminados que tienen bajo valor agregado e incluyen el planchón, la palanquilla y la barra b) los productos laminados largos como son la varilla y el alambrón que se venden principalmente en la industria de la construcción c) los productos laminados planos como son los rollos de lámina de diferentes especificaciones d) Los productos derivados que son laminados recubiertos como la lámina galvanizada, pintada, estañada, que tienen más valor agregado y se venden a la industria automotriz, línea blanca y de alimentos envasados e) la pieza de vaciado especial y forja.

La producción de semiterminados es poco deseable ya que da poco valor agregado al producto, los precios por tonelada son inferiores a los otros rubros, la contaminación por el proceso productivo es alta y la creación de empleos es baja. Sin embargo es el rubro que mayor crecimiento tuvo en estos años pasando de $12.3 \%$ en 1992 a $17.31 \%$ en 2002 .

Al analizar las exportaciones se encontró que México exporta más de 500 clases de productos dentro del grupo 72, hierro y acero. Sin embargo el principal producto que exportó a Estados Unidos es el no. 7207120050 "Productos semiterminados de hierro y acero sin alear, $0.25 \%$ carbón, rectangular, ancho cuatro veces el grueso. Este producto representaba $33.1 \%$ del total de las exportaciones siderúrgicas a Estados Unidos en 2002.

\subsection{Revisión de cada empresa y cálculo de índice de innovación}

El desempeño innovador (véase Tabla 2) se evaluó de acuerdo con la metodología del Índice Índico (Corona, 1997) evaluándose tres dimensiones: resultados, esfuerzo y capacidad. Los resultados son las innovaciones de las empresas con sus mercados, local, nacional, e internacional y sus grados de penetración. El esfuerzo evalúa los recursos asignados a las actividades de investigación y desarrollo con sus estrategias para la innovación. La capacidad o acervos tecnológicos incluyen la infraestructura y el personal con su calificación para las actividades relacionadas con la innovación. Los resultados, el esfuerzo y la capacidad se ponderan para generar el Índice Índico, el cual permite calificar a la empresa como innovadora en una escala de 10 puntos.

Hylsamex fue la siderúrgica evaluada con la mayor puntuación, ocho puntos, con respecto al desempeño innovador. Es la única que realizaba actividades de investigación y desarrollo formalmente y tenía patentes nacionales e internacionales. La empresa vende el diseño de sus plantas de hierro de reducción directa (HRD) en todo el mundo. Además, invirtió 1500 millones de dólares en tecnología; esto representa $44.4 \%$ de la inversión total de las siderúrgicas integradas en el periodo analizado. La inversión se dirigió a crear nuevas tecnologías en su planta de HRD, instalar equipo para fabricar productos como la lámina ultradelgada, producto de alta calidad y muy bajo costo. Instalaciones para fabricar aceros recubiertos, debido al incremento de su demanda en forma importante durante los 
Tabla 2

Desempeño Innovador de las empresas siderúrgicas integradas

\begin{tabular}{lcccc}
\hline Concepto & Imexa & Ahmsa & Hylsamex & Sicartsa \\
\hline Índice de innovación & 3.5 & 4.0 & 8.0 & 4.0 \\
\hline Fuente: elaboración propia utilizando la metodología del ïndice indico (Corona, 1997). &
\end{tabular}

últimos años. Lo anterior le permitió incrementar el valor agregado en su mezcla de productos ya que la lámina galvanizada se vendía a precios tres veces superiores al precio del planchón y al doble de la laminada rolada en caliente (Alvarez, 2000).

Los directivos de Ahmsa, Sicartsa e Imexa señalaron innovaciones incrementales que iban dirigidas a mejoras en el proceso con el objeto de elevar la calidad y reducir costos, sin embargo, estas innovaciones no se formalizan a través de patentes, por lo que su evaluación es inferior (Alvarez, 2000).

Imexa era la única empresa en México que fabricaba y vendia planchones (semiterminados). Esto se debe a que surtia a otras filiales del mismo grupo, ubicadas en Estados Unidos y Canadá. Sus directivos cosideraron que era una empresa muy competitiva ya que vendía su producción por anticipado, no pagaba impuestos porque al ser altamente exportadora recibía estímulos fiscales, no compraba sus materias primas en la región porque era más barato traerlas en sus barcos desde Australia y tenía un porcentaje alto de subcontratación entre sus empleados (Alvarez, 2000).

La hipótesis no se prueba porque las exportaciones que se incrementan principalmente son de productos semiterminados pero se esperaba que las em- presas altamente innovadoras tuvieran éxito en su desempeño.

En 2002 la industria presentaba una severa crisis financiera por la caida generalizada del los precios del acero, varias empresas en Estados Unidos pasaron procesos de quiebra, la inversiones en capacidad instalada de insumos como el coque, mineral de hierro y carbón se frenaron, pero de pronto a finales de 2003 se pasó de la sobreoferta de acero a la escasez, porque la República Popular China se encontraba en pleno crecimiento y demandaba grandes cantidades de acero y de materias primas para su fabricación.

China pasó de consumir 177 a 243 millones de toneladas de acero y sus importaciones pasaron de 22 a 30 millones de toneladas entre 2002 y 2004 . Los efectos fueron inmediatos para el mercado mundial; el precio de la chatarra subió $243 \%$; el coque $335 \%$; el precio del planchón $173 \%$. La industria siderúrgica tuvo un respiro y estos años han sido buenos para las empresas. Los grupos que no querían seguir en esta industria aprovecharon la prosperidad para vender sus empresas y aún las que se encontraban en suspensión de pagos tuvieron utilidades (World Steel Dymanics, 2003).

\section{Conclusiones}

En este inciso se concluye sobre tres puntos: en primer lugar la dimensión expor- 
Competitividad de la empresa industrial: una reflexión metodológica

Alvarez Medina, Lourdes

tadora no siempre deja ver claramente quién se beneficia de la actividad exportadora o si una empresa o industria son competitivas; en segundo lugar las empresas más innovadoras no siempres tienen los mejores resultados en la industria y en tercer lugar la estructura y condiciones de la industria afectan de manera importante el desempeño de las empresas.

a) La dimensión exportadora no siempre es el mejor parámetro para evaluar la competitivdad de una industria o una empresa.

De acuerdo con la literatura el parámetro más importante para evaluar competitividad son las exportaciones, pero en el caso de la siderurgia mexicana podemos ver que el incremento en exportaciones no indica una competitividad real de las empresas o de la industria.

La industria siderúrgica en México incrementó su participación en los mercados internacionales pero ganó espacio en un mercado que perdía dinamismo y además los principales productos de exportación fueron productos con bajo valor agregado. En realidad, la actuación dependía del mercado de los Estados Unidos, que compraba semiterminados y no de los esfuerzos de las empresas en México.

Se encontró que la posición competitiva de las empresas depende de otras variables que no se señalan en la literatura revisada, como pertenecer a un grupo empresarial o causas externas que afecten la demanda del producto. Además, una empresa se puede considerar a sí misma competitiva porque incrementa sus ventas en el extranjero pero al mismo tiempo no deja ningún be- neficio al país o a los individuos que en ella laboran.

Los bancos de datos para evaluar competitividad industrial como el Magic, proporcionan información que únicamente podrá interpretarse correctamente si se conocen otras condiciones de la industria; el análisis es incompleto porque no considera la diversificación de las exportaciones, el contenido tecnológico ni la base de empresas exportando.

b) Las empresas que se consideran competitivas por ser las más innovadoras no siempres tienen los mejores resultados en la indsutria.

Solamente una de las siderúrgica realizaba investigación y desarrollo, tenía un presupuesto específico y un staff con experiencia en investigación y desarrollo arriba del promedio. Sín embargo, tres años después de que se terminó el primer estudio de competitividad, la empresa no pudo recuperar su inversión debido a que los precios del acero iban a la baja y había exceso de capacidad instalada. Finalmente fue vendida a un grupo internacional.

c) La estructura y condiciones de la industria son determinantes en la competitividad de las empresas

Las condiciones de la industria resultan importantes para la competitividad empresarial. Cuando la demanda se estanca, hay exceso de capacidad instalada, el producto tiene baja diferenciación o se percibe como commodity y las utilidades disminuyen la rivalidad se exsacerva porque hay que competir por un crecimiento mínimo de la demanda y por no perder la porción de mercado que se tiene. Como se vio los esfuerzos de 
las empresas para sobrevivir se intensificaron y en ocasiones con pocos resultados. A pesar de una inversión de 1500 millones de dólares y el desarrollo de capacidade tecnológicas arriba del promedio mientras no cambiaron las condiciones de la industria una de las empresas presentó graves problemas económico. El crecimiento de la demanda permitió el incremento de los precios del acero y dio la oportunida a las empresas que venían haciendo esfuerzo poco fructiferos.

Finalmente se puede decir que la metodología para el análisis de la competitividad no puede estandarizarse, sino que es más bien un mosaico de posibilidades que debe analizarse para establecer los métodos que mejor ayuden a explicar la actuación de una empresa o una industria.

\section{Referencias Bibliográficas}

Abdel, Guillermo y Romo, David (2004). Sobre el concepto de competitividad en coord. Santiago Macias y Víctor Mirlos, Capacitación y competitividad. México, Grupo Noriega Editores, 288p.

Álvarez, Lourdes (2000). Cambio tecnológico en la industria siderúrgica mexicana integrada (1992-1999). México, Tesis doctoral, Facultad de Contaduría y Administración, UNAM, 285 p.

Álvarez, Lourdes (2002). Competitividad, comercio exterior y tecnología de la industria siderúrgica integrada en México, en coord. Nadima Simón e Isabel Rueda. Globalización y competitividad: la industria siderúrgica en México, México, Editorial Porrúa, 207$238 \mathrm{pp}$.

Aktouf, Omar (2004). "The false expectations of Michael Porter's Strategic management framework". Universidad Em- presa, vol. 3 no. 6, Bogotá, p.9-41, Octubre.

Buckley, et al. (1988). "Measures of international competitiveness: a critical survey". Journal of marketing management, UK, vol 4 no. 2, pp.175-200.

Castañon, Rosario (2003). Relaciones de los instrumentos de política industrial con la competitividad de las PYMES del sector de alimentos del D.F. y Estado de México. México, Tesis de doctoral, Facultad de Contaduría y Administración, UNAM. p.190.

Cellini, Robert y Soci, Anna (2002). "Pop Competitiveness". Banca nazionale del lavaro Quarterly Review, No. 220, pp 71-101.

Comisión económica para América Latina (CEPAL) (1999). Sistema para analizar el crecimiento del comercio internacional, (MAGIC), México.

Corona, Leonel (1997). Cien empresas innovadoras en México. México, Miguel Ángel Porrúa, p 334.

Clark, John y Guy, Ken (1998). Innovation and competitiveness: a review, Technology análisis \& Strategic management. Abingdon, sep, vol.10, no. 3, pp 363-395.

Dussel, Enrique (2003). El debate en torno de la competitividad, el concepto e implicaciones de política, en coord, Enrique Dussel, Perspectivas y retos de la competitividad en México, México, Facultad de Economía, p.20-43.

Esser, et al. (1996). "Competitividad Sistémica: nuevo desafío para las empresas y políticas". Revista de la Comisión Económica para América Latina, no. 59, Agosto, pp 39-52.

Ganzaroli, et al. (2008). "A scoreboard to evaluate cluster competitiveness: evidence from an empirical study on Emilia Romagna Region". Journal of knowledge Management, vol, $\mathrm{VI}$, no. 2, p. 63-72. 
Competitividad de la empresa industrial: una reflexión metodológica

Alvarez Medina, Lourdes

Instituto Mexicano de Ejecutivos de Finanzas (IMEF) (1995). La competitividad de la empresa mexicana, Nacional Financiera, México, 288p.

Krugman, Paul (1997). El internacionalismo moderno: la economía internacional y las mentiras de la competitividad, Grijalbo Mondadori, Barcelona, p.167.

Krugman, Paul (2000). Ricardo's. Difficult idea, consultado el 5 de abril de 2007, http://web.mit.edu/krugman/www/ricardo.htm.

Lall, Sanjaya (2000). "Competitiveness index and developing countries: an economic evaluation of the global competitiveness repot". World Development, vol, 29, No. 9, pp. 1501-1525.

Lall, Sanjaya (2003). Éxitos y fracasos industriales en un mundo en globalización, en coord. Dussel Enrique, Perspectivas y Retos de la Competitividad en México, México, Facultad de Economía, UNAM, pp 45-86.

Leontinef, Wassily (1985). Análisis económico Input-output. Barcelona, Editorial Ariel, 1a edición, 1973.

Metcalf et al. (2002). Economic development and the competitive process, Working paper, no. 34, Centre on regulation and competition, University of Manchester, UK, http://www.competitionregulation.org.uk/ consultado en abril de 2008.

Messner, Dirk y Jorge Meyer Stamer (1999). "Systemic competitiveness, Lessons from Latin America and beyond - perspectives for Eastern Europe". The European Journal of development research, Vol. 6 no. 1, pp. 89-107.

Meyer-Stamer, Jórg (2000). "Estrategias de desarrollo territorial basadas en el concepto de competitividad sistémica". El mercado de valores, México, vol. 60, No. 9, pp 48-60.
Polevnsky, Yeidckol (2003). Premisas y retos de la competitividad en México, en coord. Enrique Dussel, Perspectivas y retos de la competitividad en México, Facultad de Economía, UNAM, México, pp. 177-184.

Porter, Michael (1991). Ventaja Competitiva de las Naciones. Vergara, Buenos Aires, primera edición 1990, 535p.

Porter, Michael (2000). Estrategia competitiva: técnica para el análisis de los sectores industriales y de la competencia, primera edición en Español, 1982; CECSA, México, vigésima séptima reimpresión, $389 \mathrm{p}$.

Porter, Michael (2003). Ventaja competitiva: creación y sostenimiento de un desempeño superior. México, primera edición 1987; segunda edición, 2002; $1^{\text {a }}$ reimpresión 2003, 555 p.

Parlad, K y Gary, Hamel (1990). The core competence of the corporation, Harvard Business Review, USA, mayo-junio, pp. 79-91.

Ramírez, Delfina y Robert, Wallace (1998). Competitividad, productividad y ventaja comparativa: el enfoque de negocios de Michael Porter y el de la economía nacional de Paul Krugrman, una aplicación al caso de México. Investigación Económica, México, Vol. LVIII, No. 225, julio-septiembre, pp.17-82.

Rueda, Isabel (2000). La evolución de la industria siderúrgica en México" en Coord. Isabel Rueda y Nadima Simón, De la privatización a la crisis: el caso de Altos Hornos de México, México, Miguel Angel Porrúa Editrores, pp. 19-30.

Sanahan, Michael (2002). Steel in the 21 century: a vision for value creation, ponencia presentada en el $43^{\text {rd }}$ annual conference of the Latin American Iron and Steel Institute (ILAFA), Cancún, México, 20-23. 
Simón, Nadima (2002). Consideraciones sobre la competitividad de la industria siderúrgica en México, en Coord. Nadima Simón e Isabel Rueda. Globalización y Competitividad: la industria siderúrgica en México, México, Miguel Ángel Porrúa Editores, p. 261-273.

Villareal, René (2003). La competitividad sistémica: conceptos y condiciones en México, en Coord. Dussel Enrique, Pers- pectivas y retos de la competitividad en México, México, Facultad de Economía, UNAM, 187-208.

World Economic Forum (WEF) (http://www3. weforum.org/ consultado en octubre de 2007).

Worls Steel Dynamics (http://www.aist.org/ magazine/wsd/ consultado en octubre de 2007). 